\title{
Distribution of American Library Resources
}

$\mathrm{O}$ NE OF THE MOST frequently quoted sections of Louis R. Wilson's Geography of Reading deals with library centers in the United States. Basing his findings on the American Library Directory and several other standard reference sources issued in 1935, Wilson discovered that there were 77 centers of not over 50 miles radius (airline) containing 500,000 volumes or more. ${ }^{1}$

The Wilson study was primarily in terms of municipal centers, since the great concentrations of library resources normally are to be found in cities. At approximately the same date as the Wilson report the results of another investigation appeared, using the same sources of information, but showing the distribution of library resources by states, and more strictly limited to collections of research importance. ${ }^{2}$

How much has the situation changed in the period of about 20 years since these two studies were published? Has the number of centers increased substantially, and has their rank in relation to one another undergone any radical shifts? Are the rich states and cities getting richer and the poor poorer, or is there any

\footnotetext{
1 Louis R. Wilson, Geography of Reading (Chicago: University of Chicago Press, 1938), pp. 118-23.

2 ALA Committee on Resources of Southern Libraries, Report (Chicago: ALA, 1936), pp. 118-19. Also in Tommie Dora Barker's Libraries of the South (Chicago: ALA, 1936), Appendix A.
}

Dr. Downs is director of the library and library school, University of Illinois. He was assisted in the preparation of this article by the following graduate students of the library school: Eloise Ebert, Bernard Gill, Angelina Martinez, Peggy McCully, Furuzan Olsen, Melville Spence, Dwight Tuckwood, Howard Zandergen. evidence that library resources in different regions of the country are being equalized? These are some of the questions that the present article will attempt to answer.

The specifications used by Wilson for computing his map and table were thus stated: "In general, the area included does not cover more than 50 miles (airline) from center. When a city could be attached to more than one center, the total number of volumes in the area and transportation facilities were considered in allocating it. Public or college libraries of less than 20,000 volumes and special libraries of less than 5,000 volumes were not included. State lines were not crossed except in special cases. Centers such as Newark were maintained separately. No city was chosen as a center unless it contained one library having at least 75,000 volumes. Preference was given to state capitals, or cities in which state universities are located. Metropolitan areas were selected unless the library center would fall elsewhere."3

The second study, showing the distribution by states, included "all educational libraries with over 25,000 volumes, all public libraries over 50,000, and all special libraries of a research nature." 4

In bringing the two earlier reports up to date, the same criteria have been adhered to, in general, as in the original studies.' 5

Table I summarizes the principal data

${ }^{3}$ Wilson, op. cit., p. 119.

4 ALA Committee on Resources of Southern Libraries, op. cit., p. 119 .

${ }^{5}$ The sources used for compiling the 1955 data were: American Library Directory (New York: R. R. Bowker, 1954); American Universities and Colleges (Washington: American Council on Education, 1956); "College and University Library Statistics, 1954-55," $C R L$, XVII (1956), 56-84; U. S. Office of Education, Statistics of Public Libraries in Cities with Population of 100,000 or More: Fiscal Year 1955, Circular 471, March, 1956. 
TABle I: Library Centers, of Not Over 50 Miles Radius

\begin{tabular}{|c|c|c|c|c|c|c|}
\hline City & $\begin{array}{c}\text { Rank } \\
1955\end{array}$ & $\begin{array}{l}\text { Volumes in } \\
\text { Area, } 1955\end{array}$ & $\begin{array}{c}\text { Rank } \\
1935\end{array}$ & $\begin{array}{c}\text { Volumes in } \\
\text { Area, } 1935\end{array}$ & $\begin{array}{c}\text { Increase in } \\
\text { Volumes }\end{array}$ & $\begin{array}{l}\text { Increase } \\
\text { Per Cent }\end{array}$ \\
\hline Washington, D.C. & 1 & $25,356,917$ & 2 & $11,744,966$ & $13,611,951$ & 115.89 \\
\hline New York City, N.Y. . & 2 & $24,688,777$ & 1 & $12,910,623$ & $11,778,154$ & 91.22 \\
\hline Boston, Mass. . . . . . . . . . . & 3 & $18,334,377$ & 3 & $10,709,614$ & $7,624,763$ & 71.19 \\
\hline Chicago, Ill. . . . . . . . . . & 4 & $12,519,393$ & 4 & $6,691,144$ & $5,828,249$ & 87.10 \\
\hline Los Angeles, Calif. . . & 5 & $10,096,635$ & 5 & $6,564,016$ & $3,532,619$ & 53.82 \\
\hline Philadelphia, Pa. ... & 6 & $7,867,242$ & 6 & $4,805,252$ & $3,061,990$ & 63.72 \\
\hline San Francisco, Calif. . . & 7 & $6,901,607$ & 8 & $3,558,191$ & $3,343,416$ & 93.95 \\
\hline Cleveland, Ohio ........... & 8 & $6,140,556$ & 7 & $3,835,889$ & $2,304,667$ & 60.08 \\
\hline Newark, N.J. . . . . . . . . . . & 9 & $6,024,375$ & 9 & $2,955,827$ & $3,068,548$ & 103.82 \\
\hline New Haven, Conn. ........ & 10 & $5,330,221$ & 10 & $2,868,781$ & $2,461,440$ & 85.77 \\
\hline Minneapolis, Minn. ........ & 11 & $4,988,889$ & 13 & $2,475,322$ & $2,513,567$ & 101.57 \\
\hline Baltimore, Md. ........ & 12 & $4,857,922$ & 12 & $2,477,779$ & $2,380,143$ & 96.04 \\
\hline Albany, N.Y. . . . . . . . . . & 13 & $4,498,344$ & 19 & $1,902,574$ & $2,595,770$ & 136.41 \\
\hline Columbus, Ohio . . .... & 14 & $4,119,799$ & 20 & $1,813,637$ & $2,306,162$ & 127.12 \\
\hline Princeton, N.J. .... & 15 & $4,040,143$ & 15 & $2,150,512$ & $1,889,631$ & 87.86 \\
\hline Pittsburgh, Pa. .... & 16 & $3,937,179$ & 16 & $2,136,108$ & $1,801,071$ & 84.31 \\
\hline Cincinnati, Ohio .... & 17 & $3,750,316$ & 18 & $2,066,825$ & $1,683,491$ & 81.42 \\
\hline Detroit, Mich. ........ & 18 & $3,707,637$ & 25 & $1,404,736$ & $2,302,901$ & 163.91 \\
\hline \multicolumn{7}{|l|}{ Providence, R.I.: Fall River; } \\
\hline New Bedford, Mass. ....... & 19 & $3,646,088$ & 11 & $2,607,138$ & $1,038,950$ & 39.85 \\
\hline Hartford, Conn. ........... & 20 & $3,583,938$ & 58 & 691,217 & $2,892,721$ & 418.66 \\
\hline Chapel Hill; Durham, N.C. & 21 & $3,337,247$ & 34 & $1,091,858$ & $2,245,389$ & 205.58 \\
\hline St. Louis, Mo. . . . . . . . . . . & 22 & $3,217,632$ & 14 & $2,269,662$ & 947,970 & 41.76 \\
\hline Springfield, Mass. ...... & 23 & $3,170,131$ & 17 & $2,099,229$ & $1,070,902$ & 51.02 \\
\hline Seattle, Wash. ......... & 24 & $3,122,956$ & 36 & $1,064,818$ & $2,058,138$ & 193.23 \\
\hline Urbana, Ill. . ............ & 25 & $3,072,034$ & 33 & $1,184,928$ & $1,887,106$ & 159.24 \\
\hline San Jose; Stanford, Calif. . . . & 26 & $3,006,064$ & 22 & $1,551,791$ & $1,454,273$ & 93.68 \\
\hline Indianapolis, Ind. .......... & 27 & $3,000,499$ & 30 & $1,266,031$ & $1,734,468$ & 136.96 \\
\hline Ann Arbor, Mich. ......... & 28 & $2,884,529$ & 35 & $1,074,274$ & $1,810,255$ & 168.52 \\
\hline Buffalo, N.Y. . . . . . . . . . . & 29 & $2,809,211$ & 27 & $1,341,455$ & $1,467,756$ & 109.47 \\
\hline Denver, Colo. . . . . . . . . . . & 30 & $2,715,259$ & 32 & $1,212,159$ & $1,503,100$ & 124.00 \\
\hline Milwaukee, Wis. . . . . . . . & 31 & $2,673,066$ & 21 & $1,565,732$ & $1,107,334$ & 70.68 \\
\hline Ithaca, N.Y. . . . . . . . . . & 32 & $2,573,378$ & 31 & $1,264,920$ & $1,308,458$ & 103.39 \\
\hline Topeka, Kan. ............ & 33 & $2,505,793$ & 29 & $1,293,901$ & $1,211,892$ & 93.66 \\
\hline Lansing, Mich. . . . . . . . . . & 34 & $2,351,614$ & 45 & 797,736 & $1,553,878$ & 194.73 \\
\hline Worcester, Mass. ........ & 35 & $2,341,469$ & 28 & $1,315,636$ & $1,025,833$ & 77.96 \\
\hline Madison, Wis. . . . . . . . . . & 36 & $2,155,846$ & 26 & $1,341,899$ & 813,947 & 60.65 \\
\hline Dallas, Tex. ............... & 37 & $1,963,176$ & 70 & 570,746 & $1,392,430$ & 243.78 \\
\hline Sacramento, Calif. ........ & 38 & $1,958,441$ & 23 & $1,534,107$ & 424,334 & 27.64 \\
\hline Akron, Ohio $\ldots \ldots \ldots \ldots \ldots$ & 39 & $1,937,456$ & 44 & 799,509 & $1,137,947$ & 142.25 \\
\hline \multicolumn{7}{|l|}{ Kansas City, Mo.: } \\
\hline Kansas City, Kan. . . & 40 & $1,937,367$ & 47 & 788,837 & $1,148,530$ & 145.62 \\
\hline Austin, Tex. ............. & 41 & $1,918,420$ & 49 & 783,391 & $1,135,029$ & 144.95 \\
\hline Atlanta, Ga. ............. & 42 & $1,865,435$ & 54 & 715,842 & $1,149,593$ & 160.61 \\
\hline Des Moines, Iowa ......... & 43 & $1,851,960$ & 40 & 972,814 & 879,146 & 90.33 \\
\hline Springfield, Ill. . . . . . . . . & 44 & $1,851,364$ & 60 & 667,247 & $1,184,117$ & 177.51 \\
\hline Corvallis, Ore. ............. & 45 & $1,843,038$ & 42 & 903,154 & 939,884 & 104.09 \\
\hline Rochester, N.Y. . . . . . . . . & 46 & $1,836,532$ & 24 & $1,515,438$ & 321,094 & 21.18 \\
\hline Harrisburg, $\mathrm{Pa} . \ldots \ldots \ldots$ & 47 & $1,743,178$ & 43 & 902,802 & 840,376 & 93.02 \\
\hline Dayton, Ohio ............ & 48 & $1,637,991$ & 41 & 955,198 & 682,793 & 71.51 \\
\hline New Orleans, La. ......... & 49 & $1,625,299$ & 56 & 702,703 & 922,596 & 131.29 \\
\hline Oklahoma City, Okla. ..... & 50 & $1,550,821$ & 68 & 604,140 & 946,681 & 156.78 \\
\hline Nashville, Tenn. .... & 51 & $1,526,868$ & 46 & 791,242 & 735,626 & 93.04 \\
\hline Lexington, Ky. ...... & 52 & $1,475,022$ & 69 & 578,806 & 896,216 & 154.74 \\
\hline Richmond, Va. ........ & 53 & $1,441,119$ & 61 & 651,842 & 789,277 & 121.01 \\
\hline Baton Rouge, La. ........ & 54 & $1,415,255$ & & & & \\
\hline
\end{tabular}




\begin{tabular}{|c|c|c|c|c|c|c|}
\hline City & $\begin{array}{c}\text { Rank } \\
1955\end{array}$ & $\begin{array}{l}\text { Volumes in } \\
\text { Area, } 1955\end{array}$ & $\begin{array}{c}\text { Rank } \\
1935\end{array}$ & $\begin{array}{c}\text { Volumes in } \\
\text { Area, } 1935\end{array}$ & $\begin{array}{c}\text { Increase in } \\
\text { Volumes }\end{array}$ & $\begin{array}{l}\text { Increase } \\
\text { Per Cent }\end{array}$ \\
\hline Columbia, Mo. & 55 & $1,413,600$ & 55 & 715,829 & 697,771 & 97.48 \\
\hline Iowa City, Iowa $\ldots \ldots \ldots$ & 56 & $1,401,880$ & 59 & 679,405 & 722,475 & 106.33 \\
\hline Birmingham, Ala. ....... & 57 & $1,374,977$ & 76 & 508,381 & 866,596 & 170.66 \\
\hline Concord, N.H. . . . . . . . & 58 & $1,344,355$ & 51 & 782,818 & 561,537 & 71.77 \\
\hline Portland, Ore. ............ & 59 & $1,322,670$ & 53 & 759,320 & 563,350 & 74.17 \\
\hline Grand Rapids, Mich. ...... & 60 & $1,299,376$ & 52 & 775,680 & 523,696 & 67.52 \\
\hline Bridgeport, Conn. ....... & 61 & $1,240,975$ & 65 & 607,834 & 633,141 & 104.11 \\
\hline Lincoln, Neb. . . . . . . . . . . & 62 & $1,237,610$ & 37 & $1,031,052$ & 206,558 & 20.07 \\
\hline Houston, Tex. .......... & 63 & $1,231,685$ & & & & \\
\hline Augusta, Me. ............. & 64 & $1,230,769$ & 39 & 989,944 & 240,825 & 24.34 \\
\hline Salt Lake City, Utah ...... & 65 & $1,218,566$ & & & & \\
\hline Ft. Wayne, Ind. . . . . . . . & 66 & $1,133,926$ & & & & \\
\hline Charlottesville, Va. ....... & 67 & $1,069,935$ & & & & \\
\hline Bethlehem, Pa. .......... & 68 & $1,048,181$ & 50 & 783,274 & 264,907 & 33.84 \\
\hline Lafayette, Ind. . . . . . . . . . & 69 & $1,020,870$ & 75 & 513,855 & 507,015 & 98.63 \\
\hline San Diego, Calif. .......... & 70 & $1,010,631$ & 77 & 500,383 & 510,248 & 102.00 \\
\hline Louisville, Ky. . ......... & 71 & $1,003,900$ & 71 & 537,494 & 466,406 & 86.77 \\
\hline Fresno, Calif. . . . . . . . . . . & 72 & 971,751 & 38 & 995,404 & $(-23,653)$ & $(-2.41)$ \\
\hline Columbia, S.C. . . . . . . . & 73 & 960,247 & & & & \\
\hline Montpelier, Vt. ......... & 74 & 952,662 & 67 & 607,570 & 345,092 & 56.74 \\
\hline Utica, N.Y. . . . . . . . . & 75 & 917,844 & 74 & 514,373 & 403,471 & 78.40 \\
\hline South Bend, Ind. ......... & 76 & 911,314 & & & & \\
\hline Syracuse, N.Y. . . . . . . . . & 77 & 906,084 & 57 & 695,565 & 210,519 & 30.31 \\
\hline Oshkosh, Wis. ........... & 78 & 892,690 & & & & \\
\hline Roanoke, Va. .......... & 79 & 859,511 & & & & \\
\hline Hanover, N.H. . ........ & 80 & 831,089 & 73 & 523,641 & 307,448 & 58.58 \\
\hline Poughkeepsie, N.Y. ...... & 81 & 822,856 & 64 & 613,535 & 209,321 & 34.03 \\
\hline Toledo, Ohio . ........... & 82 & 818,293 & 63 & 620,711 & 197,582 & 31.88 \\
\hline Peoria, Ill. . .......... & 83 & 817,470 & 66 & 607,659 & 209,811 & 34.53 \\
\hline Phoenix, Ariz. ........... & 84 & 815,778 & & & & \\
\hline Knoxville, Tenn. ......... & 85 & 801,299 & & $\cdot$ & & \\
\hline Montgomery, Ala. ........ & 86 & 797,701 & & ${ }^{\circ}$ & & \\
\hline San Antonio, Tex. ........ & 87 & 782,248 & & & & \\
\hline Miami, Fla. . . . . . . . . . . & 88 & 757,906 & & & & \\
\hline Gary, Ind. . . . . . . . . & 89 & 739,501 & & & & \\
\hline Little Rock, Ark. ......... . & 90 & 736,870 & & & & \\
\hline Omaha, Neb. $\quad$............... & 91 & 724,850 & & & & \\
\hline State College, Pa. ........ & 92 & 715,896 & & & & \\
\hline Tulsa, Okla. $\quad \ldots \ldots \ldots \ldots$ & 93 & 693,836 & & & & \\
\hline Gainesville, Fla. .......... & 94 & 686,243 & & & & \\
\hline Charleston, W.Va. ........ & 95 & 678,681 & & & & \\
\hline Charlotte, N.C. . . . . . . . & 96 & 670,739 & & & & \\
\hline Athens, Ohio ............ & 97 & 664,610 & & & : & \\
\hline Greenville, S.C. . . . . . . . & 98 & 658,138 & & & & \\
\hline Rock Island, Ill. . . . . . . . . & 99 & 654,067 & & & & \\
\hline Wilmington, Del. ........ & 100 & 629,775 & & & & \\
\hline Santa Barbara, Calif. ...... & 101 & 623,121 & & $y$ & & \\
\hline Morgantown, W.Va. ....... & 102 & 596,929 & & & & \\
\hline Bakersfield, Calif. ......... & 103 & 593,277 & 62 & 641,660 & $(-48,383)$ & $(-7.47)$ \\
\hline Norfolk, Va. . . . . . . . . . . & 104 & 590,478 & & & & \\
\hline Lowell, Mass. . . . . . . . . . . . . & 105 & 580,119 & 48 & 784,843 & $(-204,724)$ & $(-26.11)$ \\
\hline Evansville, Ind. . . . . . . . . . . & 106 & 560,300 & & & & \\
\hline Erie, Pa. . . . . . . . . & 107 & 547,168 & & & & \\
\hline Wilkes-Barre, Pa. ........ & 108 & 546,564 & & & & \\
\hline Tallahassee, Fla. ......... & 109 & 537,755 & & & & \\
\hline Total $\ldots \ldots$ & & $289,355,391$ & & $138,867,606$ & $150,487,785$ & 108.36 \\
\hline
\end{tabular}


concerning library centers, i.e., the relative ranks of the centers and the number of volumes in each area in 1955 as compared to 1935, the increase in volumes, and the percentage of increase. The number of centers grew from 77 to 109 during the 20-year period, an increase of 32 . The ten leading centers in 1935 remained at the top in 1955, but there were two slight shifts in rank order: Washington, D.C., displaced New York City in the number one spot, while San Francisco pushed Cleveland from seventh to eighth place. In 1935 there were 38 centers holding over one million volumes. By 1955, the number of centers had jumped to 71 .

While the top ten were the same at

TABLE II

76 Library Centers Arranged in the Order of Their Percentage Increase, 1935 to 1955

\begin{tabular}{|c|c|c|c|}
\hline $\begin{array}{c}\text { Rank } \\
1955\end{array}$ & City & $\begin{array}{l}\text { Increase } \\
\text { Per Cent }\end{array}$ & $\begin{array}{l}\text { Rank of } \\
\text { Increase }\end{array}$ \\
\hline 20 & Hartford, Conn. ... & 418.66 & 1 \\
\hline 37 & Dallas, Tex. . . . . . . . & 243.78 & 2 \\
\hline 21 & Chapel Hill; Durham, & 205.58 & 3 \\
\hline 34 & Lansing, Mich. ........ & 194.73 & 4 \\
\hline 24 & Seattle, Wash. ...... & 193.23 & 5 \\
\hline 44 & Springfield, Ill. ..... & 177.51 & 6 \\
\hline 57 & Birmingham, Ala. ... & 170.66 & 7 \\
\hline 28 & Ann Arbor, Mich. ... & 168.52 & 8 \\
\hline 18 & Detroit, Mich. ...... & 163.91 & 9 \\
\hline 42 & Atlanta, Ga. ........... & 160.61 & 10 \\
\hline 25 & Urbana, Ill. ......... & 159.24 & 11 \\
\hline 50 & Oklahoma City, Okla. & 156.78 & 12 \\
\hline 52 & Lexington, Ky. ....... & 154.74 & 13 \\
\hline 40 & Kansas City, Mo.: & & \\
\hline & Kansas City, Kan. . & 145.62 & 14 \\
\hline 41 & Austin, Tex. ........ & 144.95 & 15 \\
\hline 39 & Akron, Ohio ......... & 142.25 & 16 \\
\hline 27 & Indianapolis, Ind. ... & 136.96 & 17 \\
\hline 13 & Albany, N.Y. ....... & 136.41 & 18 \\
\hline 49 & New Orleans, La. .... & 131.29 & 19 \\
\hline 14 & Columbus, Ohio .... & 127.12 & 20 \\
\hline 30 & Denver, Colo. ....... & 124.00 & 21 \\
\hline 53 & Richmond, Va. ..... & 121.01 & 22 \\
\hline 1 & Washington, D.C. ... & 115.89 & 23 \\
\hline 29 & Buffalo, N.Y. ......... & 109.47 & 24 \\
\hline 56 & Iowa City, Iowa ....... & 106.33 & 25 \\
\hline 61 & Bridgeport, Conn. ... & 104.11 & 26 \\
\hline 45 & Corvallis, Ore. ....... & 104.09 & 27 \\
\hline 9 & Newark, N.J. . . . . . . & 103.82 & 28 \\
\hline 32 & Ithaca, N.Y. . . . . . . & 103.39 & 29 \\
\hline 70 & San Diego, Calif. ..... & 102.00 & 30 \\
\hline 11 & Minneapolis, Minn. . . & 101.57 & 31 \\
\hline 69 & Lafayette, Ind. ........ & 98.63 & 32 \\
\hline 55 & Columbia, Mo. ...... & 97.48 & 33 \\
\hline 12 & Baltimore, Md. . & 96.04 & 34 \\
\hline 7 & San Francisco, Calif. & 93.95 & 35 \\
\hline 26 & $\begin{array}{l}\text { San Jose; Stanford, } \\
\text { Calif. } \ldots \ldots \ldots \ldots\end{array}$ & 93.68 & 36 \\
\hline 33 & Topeka, Kan. .......... & 93.66 & 37 \\
\hline 51 & Nashville, Tenn. ... & 93.04 & 38 \\
\hline
\end{tabular}

\begin{tabular}{r|l|r|c}
\hline \hline Rank & \multicolumn{1}{|c|}{ City } & $\begin{array}{c}\text { Increase } \\
\text { Per Cent }\end{array}$ & Rank of \\
Increase
\end{tabular}

* Decrease. 


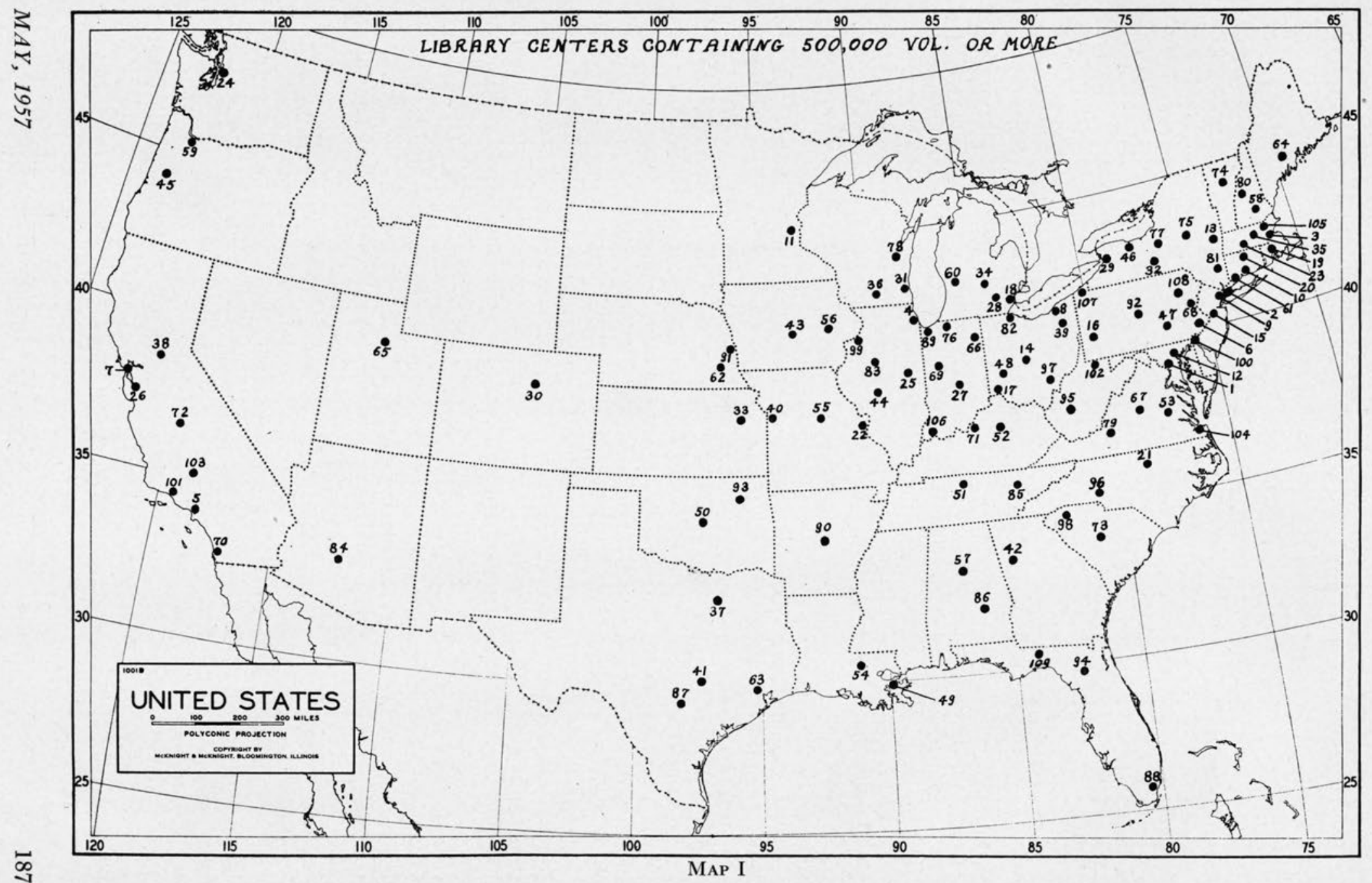


TABLE III

Comparison of Centers and Volumes in the Quarters of the Nation ForMEd BY THE 38TH PARALlel AND THE 97TH MERIDIAN

\begin{tabular}{|c|c|c|c|c|c|c|c|}
\hline \multirow[t]{2}{*}{ Quarter } & \multicolumn{2}{|c|}{ Centers } & \multicolumn{2}{|c|}{ Volumes } & \multicolumn{2}{|c|}{$\begin{array}{c}\text { Percentage } \\
\text { of Total }\end{array}$} & \multirow{2}{*}{$\begin{array}{l}\text { Increase } \\
\text { Per Cent }\end{array}$} \\
\hline & 1935 & 1955 & 1935 & 1955 & 1935 & 1955 & \\
\hline Northeast & 57 & 70 & $113,158,458$ & $223,812,024$ & 81.48 & 77.34 & 97.78 \\
\hline Southeast & 7 & 22 & $5,032,614$ & $25,092,084$ & 3.63 & 8.68 & 398.54 \\
\hline Southwest & 8 & 11 & $15,202,976$ & $28,270,353$ & 10.94 & 9.77 & 85.95 \\
\hline Northwest & 5 & 6 & $5,473,558$ & $12,180,930$ & 3.95 & 4.21 & 122.52 \\
\hline Total & 77 & 109 & $138,867,606$ & $289,355,391$ & 100.00 & 100.00 & 108.36 \\
\hline
\end{tabular}

the beginning and end of the period, their total collections were not quite so disproportionate in relation to the rest of the country in 1955 as they had been in 1935; in 1935, the ten leaders held 48 per cent of the volumes in all 77 centers, while in 1955 their holdings represented 42.6 per cent of the total volumes in the 109 centers.

Below the first ten, some striking changes in rank may be observed. For example, Hartford, Connecticut, fiftyeighth in 1935, jumped to twentieth place in 1955; Chapel Hill-Durham, North Carolina, from thirty-fourth to twenty-first; Seattle, Washington, from thirty-sixth to twenty-fourth; Dallas, Texas, from seventieth to thirty-seventh. Among the smaller centers, there appears to be little stability in rank.

Table II arranges the centers listed in the 1935 study according to their percentage of increase.

Twenty-one states gained centers during the 20-year period, accounting for a good proportion of the 32 new centers. The largest number of new centers for any one region, fifteen, appeared in the Southeast. Indiana gained four centers, and Florida, Pennsylvania, and Virginia, three each. In 1935, there were five states east of the Mississippi without a center; by 1955 , there was only one-Mississippi. West of the Mississippi, ten states lacked centers in 1935; in 1955, Arizona, Arkansas, and Utah each had a center, leaving seven western states outside the select
group_-Idaho, Montana, Nevada, New Mexico, North Dakota, South Dakota, and Wyoming.

Wilson compared the number of centers and volumes in the four quarters of the nation formed by the thirty-eighth parallel and the ninety-seventh meridian. The overwhelming lead then held by the northeast quarter was still being maintained in 1955, though the percentage of increase dropped off slightly. The story is told in Table III, and in Map I. The Northeast, which had 57 centers in 1935, had 70 in 1955, while its volume holdings grew from 113,158,458 to $223,812,024$, almost double. Of the nation's total number of volumes in the 109 centers $(289,355,391)$, only 65,543 ,367 volumes were outside the Northeast. Nevertheless, other areas had reasons for optimism and satisfaction. The most rapid expansion occurred in the Southeast, with a startling increase of nearly 400 per cent, and the Northwest grew by 122.52 per cent. The Southwest showed a small decline in relation to the other quarters.

When one turns from library centers to a consideration of the distribution of resources by states, substantially the same patterns are repeated. Conforming to the criteria for the earlier study, by the ALA Committee on Resources of Southern Libraries, only public libraries of over 50,000 volumes, educational libraries above 25,000 volumes, and spe(Continued on page 235) 


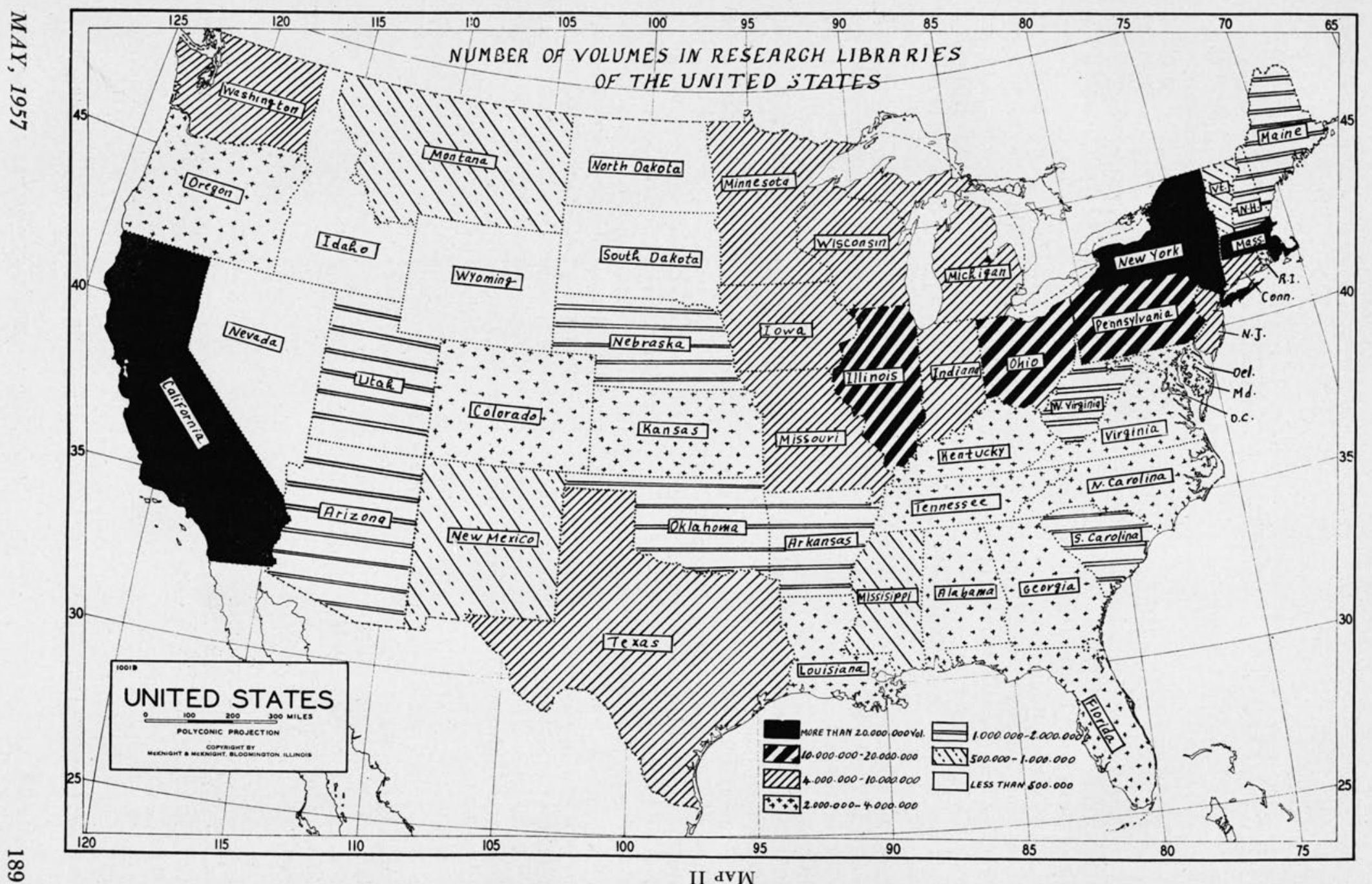




\section{Distribution of}

\section{Library Resources}

(Continued from page 188)

cial libraries of research significance have been included in the revised computations. The figures may appear somewhat inconsistent, therefore, with those noted for library centers, and it should be understood that the differences are explained by the use of different bases for tabulation. Table IV and Map II re-

TABLE IV

Number of Volumes in Research LibraRIES OF THE UNITED STATES

\begin{tabular}{|c|c|c|c|c|}
\hline $\begin{array}{l}\text { Rank } \\
1955\end{array}$ & State & $\begin{array}{c}\text { Rank } \\
1935\end{array}$ & $\underset{1935}{\text { Volumes }}$ & $\underset{1955}{\text { Volumes }}$ \\
\hline $\begin{array}{r}3 \\
4 \\
5 \\
6 \\
7 \\
8 \\
9 \\
10 \\
11 \\
12 \\
13 \\
14 \\
15 \\
16 \\
17 \\
18 \\
19 \\
20 \\
21 \\
22 \\
23 \\
24 \\
25 \\
26 \\
27 \\
28 \\
29 \\
30 \\
31 \\
32 \\
33 \\
34 \\
35 \\
36 \\
37 \\
38 \\
39 \\
40 \\
41 \\
42 \\
43 \\
44 \\
45 \\
46 \\
47 \\
48 \\
49\end{array}$ & 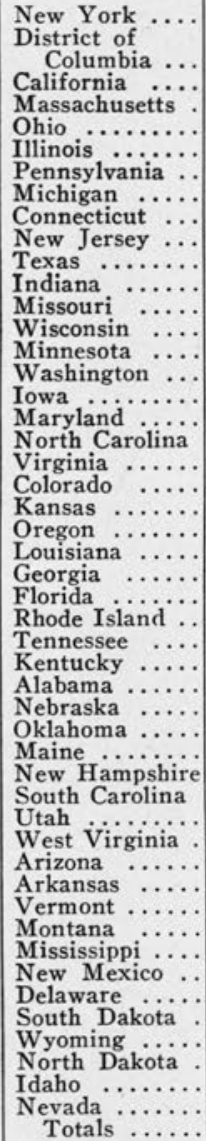 & $\begin{array}{r}1 \\
4 \\
2 \\
3 \\
5 \\
6 \\
7 \\
8 \\
9 \\
10 \\
16 \\
12 \\
11 \\
13 \\
14 \\
18 \\
15 \\
17 \\
25 \\
22 \\
23 \\
19 \\
24 \\
30 \\
31 \\
35 \\
21 \\
20 \\
27 \\
32 \\
29 \\
33 \\
26 \\
28 \\
34 \\
36 \\
39 \\
38 \\
44 \\
37 \\
40 \\
42 \\
49 \\
41 \\
45 \\
46 \\
43 \\
48 \\
47\end{array}$ & $\begin{array}{r}16,931,000 \\
10,348,000 \\
14,401,000 \\
12,944,000 \\
9,669,000 \\
8,907,000 \\
8,056,000 \\
4,427,000 \\
4,220,000 \\
4,137,000 \\
2,196,000 \\
3,345,000 \\
3,495,000 \\
3,027,000 \\
2,916,000 \\
1,665,000 \\
2,527,000 \\
2,163,000 \\
1,131,000 \\
1,552,000 \\
1,425,000 \\
1,643,000 \\
1,416,000 \\
818,000 \\
790,000 \\
447,000 \\
1,640,000 \\
1,642,000 \\
1,011,000 \\
763,000 \\
947,000 \\
720,000 \\
1,046,000 \\
948,000 \\
594,000 \\
446,000 \\
420,000 \\
425,000 \\
236,000 \\
440,000 \\
376,000 \\
267,000 \\
90,000 \\
314,000 \\
227,000 \\
187,000 \\
254,000 \\
167,000 \\
175,000 \\
137,931,000\end{array}$ & $\begin{array}{r}34,041,000 \\
25,357,000 \\
23,951,000 \\
22,951,000 \\
18,606,000 \\
17,089,000 \\
14,697,000 \\
8,988,000 \\
8,507,000 \\
8,080,000 \\
6,716,000 \\
6,520,000 \\
6,239,000 \\
6,195,000 \\
5,700,000 \\
4,415,000 \\
4,108,000 \\
3,888,000 \\
3,882,000 \\
3,684,000 \\
3,098,000 \\
3,045,000 \\
3,038,000 \\
2,797,000 \\
2,659,000 \\
2,553,000 \\
2,469,000 \\
2,412,000 \\
2,379,000 \\
2,292,000 \\
1,931,000 \\
1,902,000 \\
1,706,000 \\
1,693,000 \\
1,483,000 \\
1,330,000 \\
1,207,000 \\
1,187,000 \\
1,153,000 \\
928,000 \\
848,000 \\
828,000 \\
677,000 \\
557,000 \\
475,000 \\
470,000 \\
456,000 \\
378,000 \\
249,000 \\
279,814,000\end{array}$ \\
\hline
\end{tabular}

TABLE V

RATE OF INCREASE OF VOLUMES IN RESEARCH LIBRARIES OF THE UNITED STATES FROM 1935 To 1955

\begin{tabular}{ccc}
\hline \hline $\begin{array}{l}\text { Rank } \\
1955\end{array}$ & State & $\begin{array}{c}\text { Per Cent } \\
\text { of Increase }\end{array}$ \\
\hline
\end{tabular}

\begin{tabular}{|c|c|c|}
\hline 1 & New Mexico ............. & 652.2 \\
\hline 2 & Florida $\ldots \ldots \ldots \ldots \ldots \ldots$ & 471.1 \\
\hline 3 & $\ldots \ldots \ldots \ldots \ldots \ldots$ & 388.5 \\
\hline 4 & North Carolina ........... & 243.2 \\
\hline 5 & Louisiana $\ldots \ldots \ldots \ldots \ldots$ & 241.9 \\
\hline 6 & Georgia $\ldots \ldots \ldots \ldots \ldots$ & 236.6 \\
\hline 7 & Mississippi $\quad \ldots \ldots \ldots \ldots \ldots$ & 210.1 \\
\hline 8 & Texas $\ldots \ldots \ldots \ldots \ldots \ldots$ & 205.8 \\
\hline 9 & Alabama $\ldots \ldots \ldots \ldots \ldots \ldots$ & 200.4 \\
\hline 10 & Utah $\ldots \ldots \ldots \ldots \ldots \ldots \ldots$ & 198.2 \\
\hline 11 & West Virginia ............ & 187.4 \\
\hline 12 & Arizona $\ldots \ldots \ldots \ldots \ldots$ & 179.3 \\
\hline 13 & Oklahoma .............. & 170.9 \\
\hline 14 & Washington $\ldots \ldots \ldots \ldots \ldots$ & 165.2 \\
\hline 15 & Wyoming $\ldots \ldots \ldots \ldots \ldots$ & 151.3 \\
\hline 16 & South Carolina...$\ldots \ldots \ldots$ & 149.6 \\
\hline 17 & District of Columbia ........ & 145.0 \\
\hline 18 & Virginia $\quad \ldots \ldots \ldots \ldots \ldots$ & 137.4 \\
\hline 19 & Kentucky ............... & 135.3 \\
\hline 20 & Idaho $\ldots \ldots \ldots \ldots \ldots \ldots$ & 126.3 \\
\hline 21 & Montana . .............. & 125.5 \\
\hline 22 & Colorado ................ & 117.4 \\
\hline 23 & Oregon $\ldots \ldots \ldots \ldots \ldots \ldots$ & 114.5 \\
\hline 24 & Vermont $\ldots \ldots \ldots \ldots \ldots$ & 110.9 \\
\hline 25 & South Dakota ............ & 109.3 \\
\hline 26 & Wisconsin $\ldots \ldots \ldots \ldots \ldots$ & 104.6 \\
\hline 27 & Nebraska ............... & 103.9 \\
\hline 28 & Michigan $\quad . \ldots \ldots \ldots \ldots \ldots$ & 103.0 \\
\hline 29 & Connecticut $\ldots \ldots \ldots \ldots \ldots$ & 101.6 \\
\hline 30 & New York ............... & 101.1 \\
\hline 31 & Minnesota ............... & 95.5 \\
\hline 32 & New Jersey ............... & 95.3 \\
\hline 33 & Indiana $\ldots \ldots \ldots \ldots \ldots$ & 94.9 \\
\hline 34 & Ohio $\ldots \ldots \ldots \ldots \ldots \ldots$ & 92.4 \\
\hline 35 & Illinois $\ldots \ldots \ldots \ldots \ldots \ldots$ & 91.9 \\
\hline 36 & Kansas . . . . . . . . . . . & 85.3 \\
\hline 37 & Pennsylvania $\ldots \ldots \ldots \ldots \ldots$ & 82.4 \\
\hline 38 & Maryland ... & 79.7 \\
\hline 39 & North Dakota ............ & 79.5 \\
\hline 40 & New Hampshire ........... & 78.6 \\
\hline 41 & Missouri $\quad \ldots \ldots \ldots \ldots \ldots$ & 78.5 \\
\hline 42 & Delaware .... & 77.4 \\
\hline 43 & Massachusetts $\ldots \ldots \ldots \ldots \ldots$ & 77.3 \\
\hline 44 & California $\ldots \ldots \ldots \ldots \ldots \ldots$ & 66.3 \\
\hline 45 & Maine $\ldots \ldots \ldots \ldots \ldots \ldots$ & 63.1 \\
\hline 46 & Iowa $\ldots \ldots \ldots \ldots \ldots \ldots \ldots$ & 62.6 \\
\hline 47 & Rhode Island . . . . . . . . & 50.5 \\
\hline 48 & Tennessee ... & 46.9 \\
\hline \multirow[t]{2}{*}{49} & Nevada . . & 42.2 \\
\hline & National average ... & 102.1 \\
\hline
\end{tabular}

